\title{
Performance of the ARIANNA pilot array, and implications for the next generation of UHE neutrino detectors
}

\author{
Christopher Persichilli* for the ARIANNA Collaboration ${ }^{\dagger}$ \\ University of California, Irvine, USA \\ E-mail: cpersich@uci.edu
}

\begin{abstract}
The seven-station test-bed for the ARIANNA ultra-high energy (UHE) neutrino telescope, the Hexagonal Radio Array (HRA), was completed during the 2014-2015 Antarctic summer season. In the more than four years since, the ARIANNA pilot array has demonstrated remarkable resilience and stability, surviving multiple Antarctic winters while operating with a typical $90 \%$ uptime during the summer months, while the ARIANNA data acquisition system has proven to be capable and cost/power efficient. A combined analysis of 4.5 years of data from the pilot array will be presented. The neutrino search yielded no candidate events, with a signal efficiency of 79\%, providing an updated limit for the diffuse UHE neutrino flux of $E^{2} \Phi=1.7 \times 10^{-6}$ $\mathrm{GeV} \mathrm{cm}^{-2} \mathrm{~s}^{-1} \mathrm{sr}^{-1}$ at a neutrino energy of $10^{18} \mathrm{eV}$. Data from the operation of the ARIANNA pilot array will inform the design of the next generation of radio neutrino detectors, which will be capable of probing all but the most pessimistic models of the GZK neutrino flux. It will also be shown that such detectors will have an important role to play in the multi-messenger detection of point sources.
\end{abstract}

36th International Cosmic Ray Conference -ICRC2019-

July 24th - August 1st, 2019

Madison, WI, U.S.A.

\footnotetext{
* Speaker.

${ }^{\dagger}$ for collaboration list see PoS(ICRC2019)1177
} 


\section{Introduction}

Determining the source, or combination of sources, which contribute to the measured flux of ultra-high energy (UHE) cosmic rays, which have been measured with energies in excess of $10^{20} \mathrm{eV}$ [1], is at the heart of one of the most fundamental unsolved problems in astronomy. Radio-based detectors, such as ARIANNA, leverage the $\mathscr{O}(1 \mathrm{~km})$ attenuation lengths for radio frequencies in cold ice to search for the characteristic Askaryan pulses generated by neutrino induced particle showers [2,3]. Such designs can economically instrument large volumes at modest cost, and will be a valuable tool for the detection of UHE neutrinos.

The intriguing correlation of neutrinos measured in IceCube with the blazar, TXS 0506+056 $[4,5]$, prompted renewed interest in the role of neutrinos in multi-messenger astronomy. Theories of UHE cosmic ray production predict a deep relationship between gamma-rays, astrophysical neutrinos, and UHE charged cosmic rays [6]. In addition to constraining the diffuse cosmogenic neutrino flux, the sensitivity of radio-based neutrino detectors to astrophysical neutrinos from luminous transient events allows for exciting contributions to multi-messenger astronomy and the potential to study source objects via light, gravitational waves, and neutrinos.

\section{The ARIANNA detector}

The ARIANNA pilot Hexagonal Radio Array (HRA) consists of seven neutrino detector stations installed at the Moore's Bay site on the Ross Ice Shelf. Each station contains a set of four LPDA antennas installed just below the snow surface, pointing downward into the Ice Shelf. The ice/seawater boundary below Moore's Bay acts as a highly efficient mirror at radio frequencies, which significantly increases the effective volume of the detector, and provides fairly uniform sensitivity across the entire upward sky. The Moore's Bay site also has the unique advantages of an extremely radio quiet environment, owing to shielding from nearby mountain ranges, while also being close enough to McMurdo Station to take advantage of surface logistics.

Each station is a self-contained and autonomous detector, powered by individual solar panels, and able to transfer event data to the US in near real-time through a combination of long range $2.4 \mathrm{GHz}$ wireless ethernet and Iridium satellite communication systems. This design allows for the deployment of ARIANNA stations in at any location with ice of sufficient quality, without the need for infrastructure development.

In December 2015 the battery systems on all HRA stations were upgraded, leading to improved livetime and stability for the array. When the array is configured to maximize livetime, ARIANNA stations have been shown to achieve sustained operational efficiencies of 90\% (figure 1).

The current pilot array stations are based on the Synchronous Sampling plus Triggering (SST) chip developed [7]. Each event trigger records a $128 \mathrm{~ns}$ record at $2 \mathrm{GSa} / \mathrm{s}$. The SST employs a multi-stage coincidence trigger. The first stage requires a $5 \mathrm{~ns}$ coincidence between high and low threshold crossing on a single input channel. The second stage requires that two or more channels trigger within a $30 \mathrm{~ns}$ window, taking advantage of that parallel pairs of LPDA's see largely the same signal. This schema achieves a $\mathscr{O}(\mathrm{mHz})$ thermal trigger rates at a trigger threshold of $4 \sigma$ above thermal $V_{R M S}$ amplitude, which allows for real-time data transfer. Events which are not 
transferred in real-time can be transferred on demand at a later date, or physically recovered from internal storage.
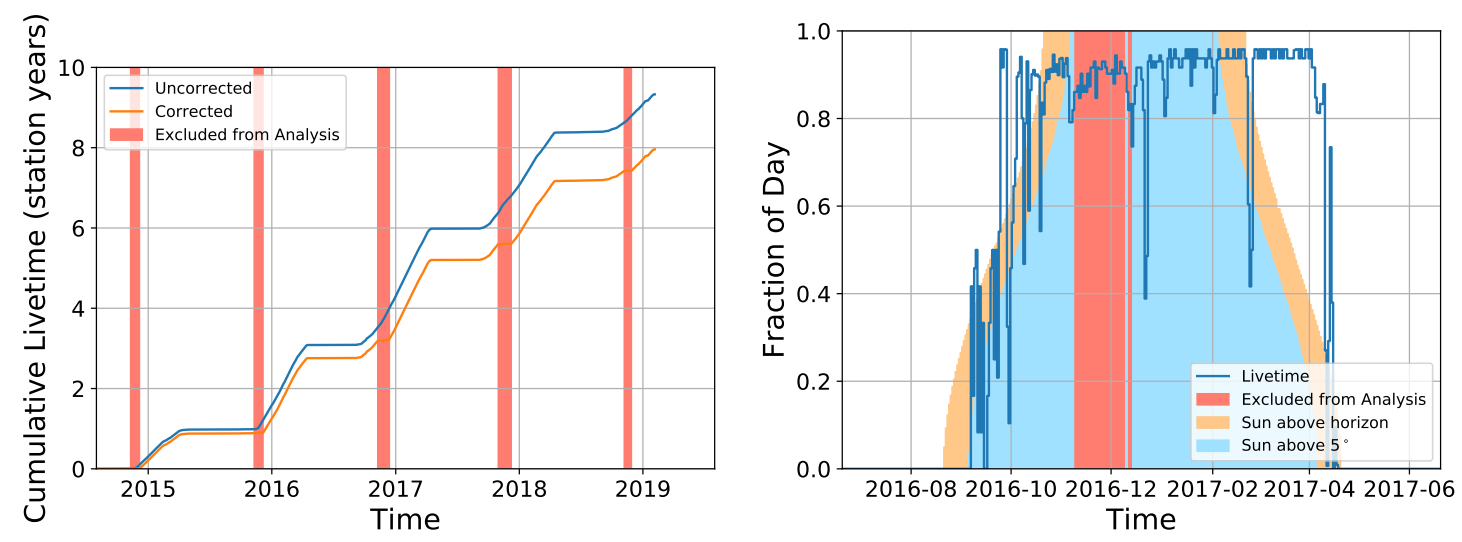

Figure 1: (left) Cumulative livetime for all SST based HRA stations since deployment. The corrected curve represents the livetime used for analysis, as discussed in section 4. Since the HRA stations rely on solar power, they shut down during the winter and do not accrue livetime. (right) The livetime per day for an HRA station. Configurations are tuned to maximize livetime after the end of the field season (red band), after-which stations typically show livetimes $>90 \%$. Shaded regions show fractions of the day that the sun is above the horizon, and more than $5^{\circ}$ above the horizon.

\section{Simulation of Neutrino Signal}

In order to generate a population of simulated neutrinos for analysis, one billion neutrino interactions were simulated in the ShelfMC Monte Carlo [8] with a GZK energy spectrum [9], using a detector configuration matching the currently deployed ARIANNA HRA stations in Moore's Bay. This produced a sample of approximately 1.3 million events which triggered the simulated station at a $4 \sigma$ level above the thermal noise $V_{R M S}$.

The time-domain signal is produced by generating waveform templates which incorporate the response of the antennas and amplifiers, according to the procedure in [10]. Two versions of the ARIANNA amplifiers (100 and 200 series) are in use in the pilot array, which have slight differences in their impulse response. To account for these differences, separate templates are constructed for each type of amplifier, and the stations which contain each type are analyzed separately. The simulated event geometry is used to select the most appropriate template for each simulated neutrino, which is scaled to the correct amplitude and added to finite bandwidth thermal noise. The simulated signal is converted to the same data format, and processed by the exact same analysis, as the triggered events.

\section{Analysis of Triggered Events}

A template matching procedure is used to distinguish signal from background. A Pearson correlation coefficient $\chi$ used to compare a specific reference template to the measured signal on each channel of a triggered event. Values of $\chi$ range from 0 (no correlation) to 1 (identically 
similar). After calculating $\chi$ for each channel, we combine the results into a single parameter per event, $\chi_{\text {ave }}$, which is the greater of the two averages between co-polarized pairs of LPDA's. High values of $\chi_{\text {ave }}$ essentially imposes a condition that pairs of antennas see similar signals, which is expected for neutrino signals.

By also considering the signal amplitude, it is possible to leverage the general behavior that a high signal-to-noise ratio (SNR) event is likely to have a greater value of $\chi_{\text {ave }}$ than for a low SNR event. In this text, the SNR of an event will be defined as $V_{P T P} / 2 \sigma$, where $V_{P T P}$ is the maximum peak to peak amplitude across all channels, and $\sigma$ is the root-mean-squared voltage of min-bias noise events.

We create a 2D parameter space using $\chi_{\text {ave }}$ and SNR in which we will define a signal region for this analysis. The distribution of the simulated neutrino signal in this space is shown in figure 2 .
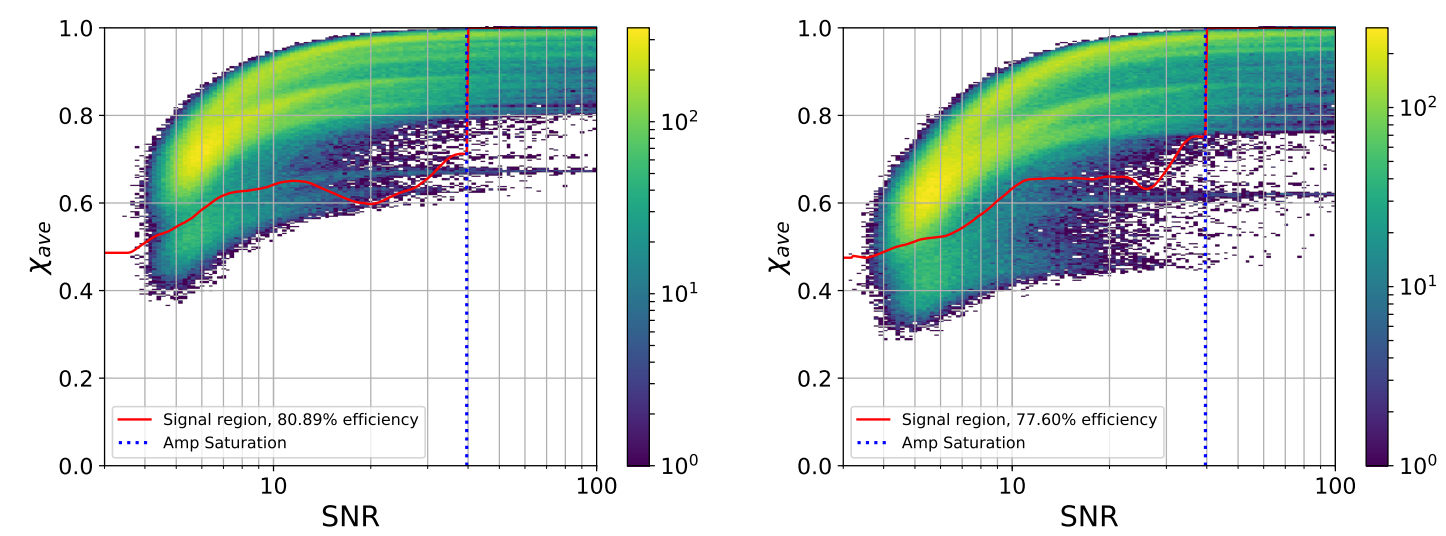

Figure 2: Distribution of simulated neutrino signal in $\chi_{\text {ave }}$ vs SNR. Stations with series 100 amps (left) and series 200 amps (right) are analyzed separately, as discussed in section 3. Notice that larger values of SNR correspond to larger values in $\chi_{\text {ave }}$, since lower SNR leads to worse cross-correlation to the reference template. The blue vertical line represents a $800 \mathrm{mv}$ cutoff where the amplifiers would be strongly non-linear. The red line represents the lower bound of the signal region, which is discussed in section 4.

We can define a lower bound for a neutrino signal region within this parameter space such that the expected number of background events will be 0.5 across all stations for the entire data set. Two versions of the ARIANNA amplifiers (100 and 200 series) are employed in the pilot array. Stations with different amplifier models will be analyzed separately, in order to account for differences in hardware response.

The data for this analysis includes all triggered events collected from the current SST based pilot array stations from after their deployment (beginning December $8^{\text {th }}, 2014$ ) through February $5^{\text {th }}, 2019$. The total livetime for this analysis is 7.96 station years (figure 1), which excludes times when the Moore's Bay site was occupied, as well as some periods on Dec 11-13 ${ }^{\text {th }}, 2016$ during the operation or ANITA's HiCal pulser [11], which was observed by the HRA.

Events are binned according to a sliding 0.2 decade window in $\log S N R$. The expected number of background events in a bin is taken to be the total number of expected events (0.5) weighted by the fraction of neutrino events in the bin, which effectively produces a curve of constant signal to background ratio. In order to the calculate the $\chi_{\text {ave }}$ cutoff, the tail of the cumulative distribution 
is fit to a power law, and extrapolated to the desired number of background events in the bin. The resulting curve is then smoothed by a second order Savitzky-Golay filter. Some technical details and a graphical illustration of this process are shown in figure 3. An expected number of 0.5 background events within the full data set results in a neutrino signal efficiency of $79 \%$.

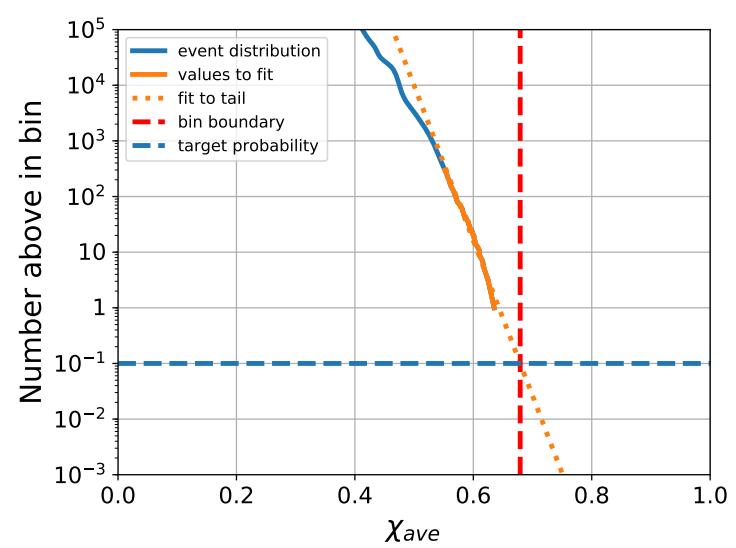

Figure 3: An illustration of the procedure for calculating the signal region boundary for a particular sliding SNR bin. A power law is fit to the 300 highest $\chi_{\text {ave }}$ events in the bin (the single highest value event is excluded from the fit in order to limit the effect of outliers), and extrapolated to intersect the desired background event probability, yielding the signal region lower bound value of $\chi_{\text {ave }}$.

The $\chi_{\text {ave }}$ - SNR distribution of the measured data set is presented in Fig. 4. No candidate neutrino events were observed in the signal region.
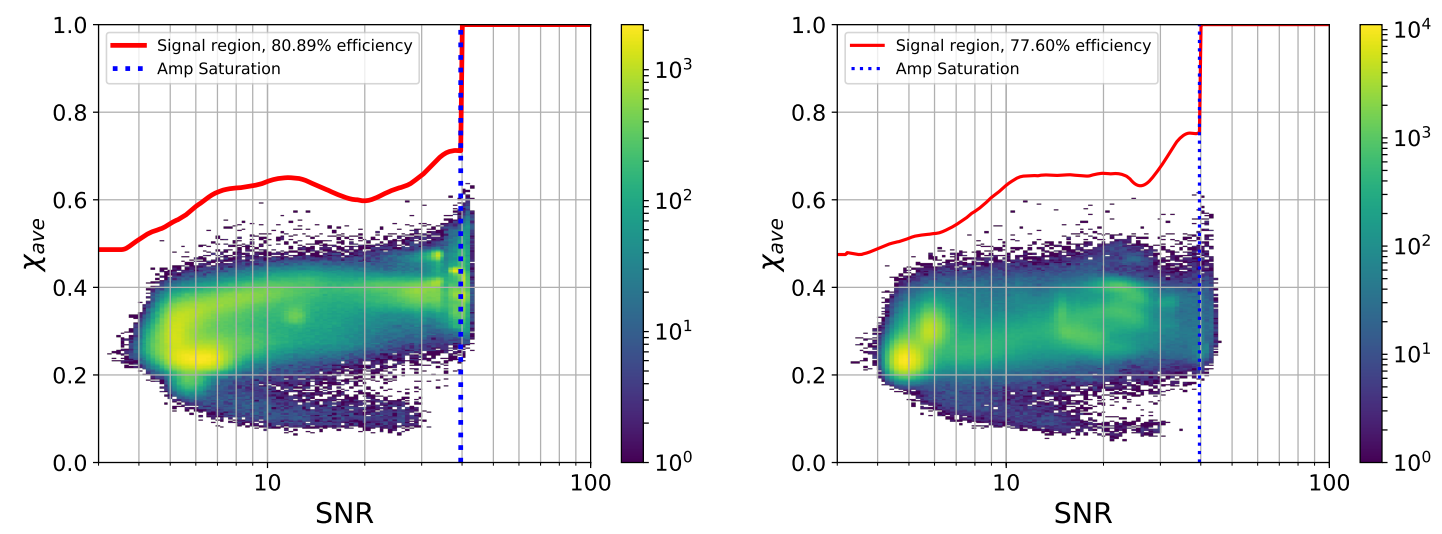

Figure 4: Distribution of triggered events in $\chi_{a v e}$ Vs SNR. Stations with series 100 amps (left) and series 200 amps (right) are analyzed separately, as discussed in section 3. The blue horizontal line represents a $800 \mathrm{mv}$ where a noticeable pile-up effect is observed due to amp clipping. The red line represents the lower bound of the signal region, which is discussed in section 4 . No triggered events remain in the signal region.

\section{Diffuse Flux Limits}

In the absence of observed events in the signal region, a model independent $90 \%$ confidence upper limit on the diffuse neutrino flux is given by, 


$$
E^{2} \Phi(E) \leq \frac{F C_{90} E L(E)}{\ln 10 d \log E V_{e f f} \Omega \sum_{i}\left(\varepsilon_{i} t_{i}\right)}
$$

where $F C_{90}=2.44$ is the Feldman Cousins $90 \%$ confidence upper limit per decade-wide bin, $E$ is the neutrino energy, $d \log E=1$ is the bin width in decades, $V_{e f f} \Omega$ is the effective volume of a single ARIANNA station averaged over all flavors, $L$ is the water equivalent interaction length calculated using the cross section in [12], and $t_{i}$ and $\varepsilon$ are, respectively, the total livetime and the analysis efficiency for each station. The result of this calculation for the ARIANNA pilot array is shown in figure 5.
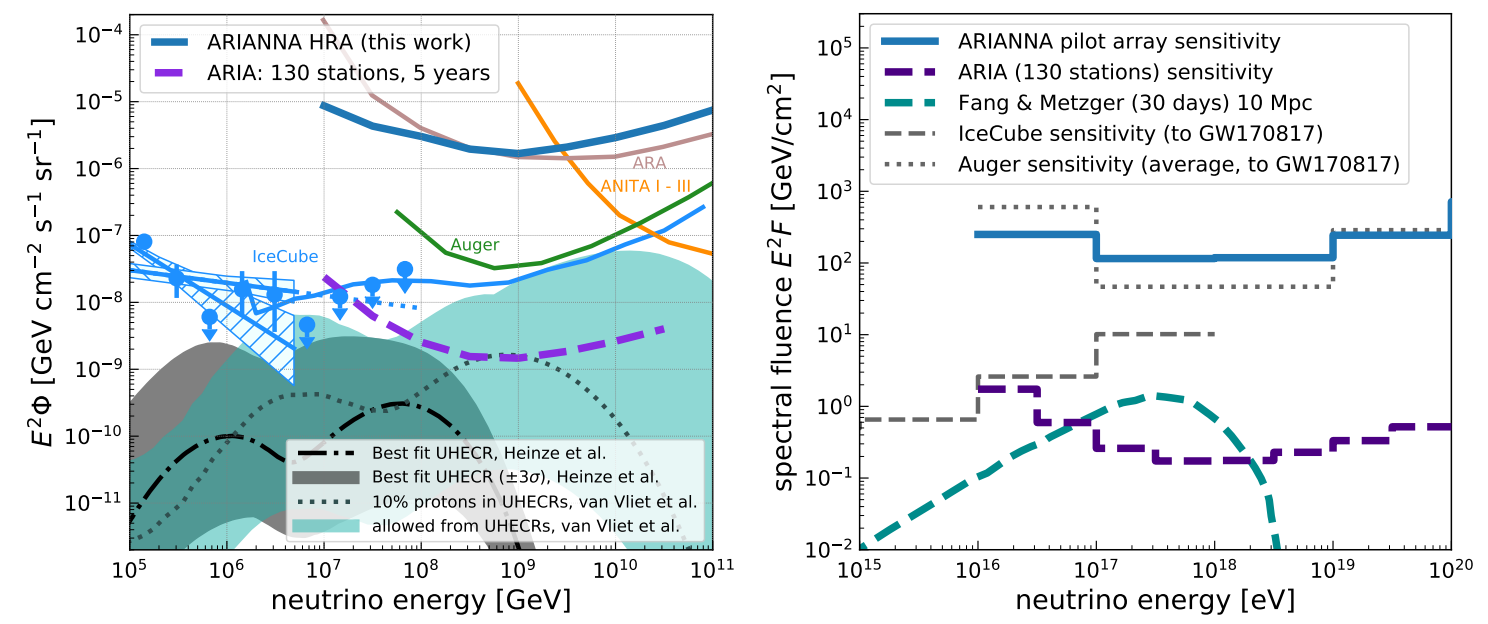

Figure 5: (left) Diffuse flux limit for the ARIANNA HRA on a sliding decade wide energy bin. For details of the calculation see section 5. (right) Sensitivity of the ARIANNA pilot array to transient sources. Figures adopted from [13] (see references therein, as well as [14], for the shown limits and spectra).

\section{Discussion and Outlook}

We have demonstrated that a template matching procedure is capable of rejecting all observed backgrounds over the operation of the HRA, while maintaining a combined signal efficiency of $79 \%$ at a $4 \sigma$ trigger threshold in amplitude. Future detectors will make use of more advanced triggering and analysis techniques in order to push the sensitivity threshold lower, and increase the effective volume per station. In addition, the development of new wind power systems for Antarctic environments offers the potential to significantly increase the livetime of remote autonomous stations through the winter, without the need for investment in power infrastructure [15].

The operation of the ARIANNA pilot array at Moore's Bay, since it's completion in December 2014, has provided a robust proof of concept for an autonomous, radio-based UHE neutrino detector. Iterative improvements in data acquisition and power systems since the first ARIANNA prototypes [16] and initial ARIANNA stations [7] have reduced cost and power consumption, while improving reliability and livetime. Practical experience gained through the deployment and operation of the ARIANNA pilot array will provide crucial insight for the development of future experimental designs. 
Using the knowledge gained through the operation of the ARIANNA pilot array, it is possible to make informed projections of the sensitivity of a future full-scale detector. A proposed design for an optimized surface array (ARIA) is discussed in [13], and the projected flux limit is shown in figure 5 for comparison. This design expands on the basic ARIANNA station layout by including a $15 \mathrm{~m}$ vertically oriented string of dipoles, as well as other design changes which improve both the sensitivity of the detector, as well as the ability to perform a precise reconstruction of neutrino event properties, which will be vitally important in a first detection of UHE neutrinos [17].

Since each ARIANNA station serves as a autonomous and self-contained detector, this platform can be deployed at various sites in order to attain full sky coverage. Near-surface detectors allow for the easy installation of high-gain antennas in any polarization, providing increased information for precise event reconstruction [17]. In particular, a precise measurement of neutrino arrival direction will enable real-time alerts to be sent out to the community, allowing for directed multi-messenger follow up. Additionally, the large effective volume of radio-based detectors makes them highly sensitive to explosive events, e.g. the sensitivity of the ARIANNA pilot array is comparable to Auger for objects within the field of view (figure 5), which is effectively the entire upward sky. This uniform sensitivity, which comes as a property of Moore's bay's ice/sea boundary, is of significant benefit in the hopeful detection of rare transient events.

\section{Acknowledgements}

We are grateful to the U.S. National Science Foundation-Office of Polar Programs, the U.S. National Science Foundation-Physics Division (grant NSF-1607719) for granting the ARIANNA array at Moore's Bay. In particular, we are grateful for the excellent support of the McMurdo region staff, without which the scientific mission in Antarctica would not be possible.

We acknowledge funding from the German research foundation (DFG) under grants GL 914/11 and NE 2031/2-1 and the Taiwan Ministry of Science and Technology. HB acknowledge support from the Swedish Government strategic program Stand Up for Energy. EU acknowledge support from the Uppsala university Vice-Chancellor's travel grant (sponsored by the Knut and Alice Wallenberg Foundation) and the C.F. Liljewalch travel scholarships. DB and ANo acknowledge support from the MEPhI Academic Excellence Project (Contract No. 02.a03.21.0005) and the Megagrant 2013 program of Russia, via agreement 14.12.31.0006 from 24.06.2013.

\section{References}

[1] AUGER collaboration, The cosmic ray energy spectrum measured using the pierre auger observatory, in Proceedings of 35th International Cosmic Ray Conference - PoS(ICRC2017), Sissa Medialab, Aug., 2017, DOI.

[2] G. A. Askar'yan, Coherent Radio Emission from Cosmic Showers in Air and in Dense Media, Soviet Journal of Experimental and Theoretical Physics 21 (1965) 658.

[3] ANITA collaboration, Observations of the askaryan effect in ice, Physical Review Letters 99 (2007) .

[4] The IceCube Collaboration, et al., Multimessenger observations of a flaring blazar coincident with high-energy neutrino IceCube-170922a, Science (2018) eaat1378. 
[5] The IceCube Collaboration, Neutrino emission from the direction of the blazar TXS 0506+056 prior to the IceCube-170922 a alert, Science (2018) eaat2890.

[6] M. Ahlers and F. Halzen, Opening a new window onto the universe with IceCube, Progress in Particle and Nuclear Physics 102 (2018) 73.

[7] ARIANNA collaboration, Design and Performance of the ARIANNA Hexagonal Radio Array Systems, IEEE Transactions on Nuclear Science (2015) [1410.7369].

[8] C. Persichilli, Performance and Simulation of the ARIANNA Pilot Array, with Implications for Future Ultra-high Energy Neutrino Astronomy, Ph.D. thesis, University of California, Irvine, 2018.

[9] R. Engel, D. Seckel and T. Stanev, Neutrinos from propagation of ultrahigh energy protons, Physical Review D 64 (2001).

[10] ARIANNA collaboration, Time-domain response of the ARIANNA detector, Astroparticle Physics 62 (2015) 139 [1406.0820].

[11] S. Prohira, A. Novikov, D. Besson, K. Ratzlaff, J. Stockham, M. Stockham et al., Hical 2: An instrument designed for calibration of the anita experiment and for antarctic surface reflectivity measurements, Nuclear Instruments and Methods in Physics Research Section A: Accelerators, Spectrometers, Detectors and Associated Equipment 918 (2019) 60 .

[12] A. Connolly et al., Calculation of high energy neutrino-nucleon cross sections and uncertainties using the martin-stirling-thorne-watt parton distribution functions and implications for future experiments, Physical Review D 83 (2011).

[13] A. Anker, S. Barwick, H. Bernhoff, D. Besson, N. Bingefors, G. Gaswint et al., Targeting ultra-high energy neutrinos with the arianna experiment, Advances in Space Research (2019) .

[14] ARA collaboration, Performance of two askaryan radio array stations and first results in the search for ultrahigh energy neutrinos, Phys. Rev. D 93 (2016) 082003.

[15] A. Nelles for the ARIANNA collaboration, A wind-turbine for autonomous stations for radio detection of neutrinos experiment, PoS(ICRC2019)968 (these proceedings) (2019) .

[16] L. Gerhardt, S. Klein, T. Stezelberger, S. Barwick, K. Dookayka, J. Hanson et al., A prototype station for arianna: A detector for cosmic neutrinos, Nuclear Instruments and Methods in Physics Research Section A: Accelerators, Spectrometers, Detectors and Associated Equipment 624 (2010) 85 .

[17] C. Glaser for the ARIANNA collaboration, Neutrino direction and energy resolution of askaryan detectors, PoS(ICRC2019)899 (these proceedings) (2019) . 